\title{
Deployment Analysis of a Simple Tape-Spring Hinge Using Probabilistic Methods
}

\author{
Karen H. Lyle ${ }^{*}$ and Lucas G. Horta ${ }^{\dagger}$ \\ NASA Langley Research Center, Hampton VA 23681
}

\begin{abstract}
Acceptance of new deployable structures architectures and concepts requires validated design methods to minimize the expense involved with technology validation flight testing. Deployable concepts for large lightweight spacecraft include booms, antennae, and masts. This paper explores the implementation of probabilistic methods in the design process for the deployment of a strain-energy mechanism, specifically a simple tape-spring hinge. Strain-energy mechanisms are attractive for deployment in very lightweight systems because they do not require the added mass and complexity associated with motors and controllers. However, designers are hesitant to include free deployment, strain-energy mechanisms because of the potential for uncontrolled behavior. In the example presented here, the tapespring cross-sectional dimensions have been varied and a target displacement during deployment has been selected as the design metric. Specifically, the tape-spring should reach the final position in the shortest time with the minimal amount of overshoot and oscillations. Surrogate models have been used to reduce computational expense. Parameter values to achieve the target response have been computed and used to demonstrate the approach. Based on these results, the application of probabilistic methods for design of a tape-spring hinge has shown promise as a means of designing strain-energy components for more complex space concepts.
\end{abstract}

\section{Introduction}

$\mathrm{H}$ IGH reliability, deployable booms, masts, and antennae have been and will continue to be in demand for all classes of space applications. CubeSat class missions are driving requirements for low weight, mechanically simple, strain-energy deployed structures [1]. Crewed solar-electric propulsion vehicles will require very large, robust, deployable solar arrays [2]. Astronomy and physics applications for very large deployable apertures beyond the James Webb Space Telescope drive requirements with added demands of high deployment precision and stability [3]. It is particularly challenging to demonstrate deployment reliability on the ground for mission critical, ultra-lightweight deployed membrane structures, including sunshades, exoplanet starshade occulters, and solar sails, Such concepts will require validation through analysis. The current design verification and validation practice is based on full-scale testing for expandable structures. Additionally, flight testing for the purpose of technology validation is often expensive. Fortunately, significant advances have occurred in the numerical simulation of highly nonlinear dynamic systems. For example, simulations can incorporate structural complexities, such as geometrically accurate models and advanced material models to include nonlinear stress-strain behaviors, laminated composites, and material failure [4].

The tape-spring hinge problem was selected for this example because it addresses several design challenges. 1) Tape-spring hinges have an engineering heritage and continue to be used but formal design approaches do not exist. 2) Strain-energy mechanisms exhibit transient dynamic, geometrically nonlinear behavior; therefore models must incorporate these basic phenomena. 3) Designs of such systems require computationally efficient modeling tools. These types of strain-energy components have been incorporated in spacecraft since Sputnik and continue with the recent use of strain-energy joints in the MARSIS antenna [5] on the Mars Express spacecraft. However, detailed computational tools to analyze the deployment of such systems have more recently emerged. References [6]-[11] have examples that illustrate strain-energy mechanism applications. These publications provide detailed experimental, analytical, and numerical insights about the quasi-static folding and initial deployment of strainenergy mechanisms. Computationally efficient models are also critical to enable completion of numerous

\footnotetext{
${ }^{*}$ Research Aerospace Engineer, Structural Dynamics Branch/MS 230.

${ }^{\dagger}$ Research Aerospace Engineer, Structural Dynamics Branch/MS 230, and AIAA Associate Fellow.
} 
simulations in cases where numerous executions of nonlinear, transient dynamics simulations are needed to verify the design. This study concentrates on extending the work in Ref. [12] by implementing probabilistic methods in the design process. Previously, the simulation results for tape-spring folding [12] were compared with data provided in Ref. [13]. (Ref. [13] did not contain free deployment data.)

Probabilistic analysis (PA) methods are often used to study parameter variability during the design process for the purposes of assessing reliability and robustness. In these cases parameter variations and their representation are selected based on expected parameter excursions from the as-built system. However, in this study PA tools are used to conduct parametric studies where the uncertainty model description represents a design space, as opposed to the uncertainty space. It is easy to lose sight of the fact that for most nonlinear, transient-dynamic design problems, brute force approaches are often impractical, and more sophisticated methods may be required to optimally use the results from a relatively small number of simulations. For example, thousands of simulations using a Monte Carlo approach are typically not feasible. As the number of parameters, number of responses, and runtimes increase, particularly in conjunction with a time-varying metric, a more streamlined and systematic approach may be required. One option is the implementation of probabilistic methods to provide additional insights. Reference [14] gives an example for an aeronautics crash application.

This paper begins with a brief description of the finite element model (FEM) followed by a short description of the probabilistic modeling approach used and a discussion of the results. Within the discussion of the results, the probabilistic design process description is divided into two phases: Phase A-Assessing parameter importance and Phase B-Parameter selection process to produce target results. The discussion of the results also includes the design goals and a design to meet the goals. Finally, concluding remarks provide general comments about the approach and findings.

\section{Description of Modeling and Analyses}

\section{A. Finite Element Model (FEM)}

Figure 1 shows the geometry and sample mesh used to model the tape-spring along with associated folding nomenclature, i.e., opposite-sense (OS) and same-sense (SS). Also included in the figure is a description of the boundary conditions and enforced end rotations. At each end of the tape-spring, a rigid body has been formed by connecting the nodes at the end with an extra node located at the geometric center of the arc, denoted as Node A and Node B. An equal but opposite enforced rotation has been applied at Nodes A and B. Prior to completion of the probabilistic design process and to verify numerical stability, an extensive review of several simulation responses was performed. These responses include tape-spring kinetic and internal energies, end rotations, and end displacements. For example, the internal energy at release can be viewed as an intermediate "initial condition" for the deployment. After much review, the controlled and prescribed resultant displacement of the free end (Node B) was selected as an appropriate evaluation and design response. The tape material properties are copper-beryllium, a common spacecraft alloy. Table 1 provides the specifics about the tape.

Results reported here were focused on varying geometric parameters describing the tape-spring hinge cross section (Fig. 2). A finite element model with 100 elements along the $0.2 \mathrm{~m}$ length of the tape and 20 elements along the tape arc (for a total of 2,000 elements) was used. The simulations were executed in LS-Dyna, ${ }^{\mathrm{TM}}$ a commercial, general-purpose, nonlinear, transient dynamics, finite element code widely used in crash and impact application [15]. Each simulation required approximately $1 \frac{1}{2}$ to 2 hours using four processors to compute the 0.5 seconds of folding and free deployment. Execution time was inversely proportional to the arc length and radius.

\section{B. Probabilistic Analysis Approach}

Numerical values for each of the parameters identified in Figure 2 are assumed to be equally likely (i.e., to have uniform distribution) within the ranges specified in Table 2. Eighty simulations were performed for both OS and SS folding configurations. These two general folding cases can be easily tested with a simple tape spring, and OS and SS configurations are frequently combined for hinge deployment stability. For this implementation, MATLAB scripts were written to control the simulation execution and to store simulation data for all cases.

Several approaches exist to conduct Analysis of Variance (ANOVA) for global parameter sensitivity estimates. In most cases, design of experiments sampling of the parameter space is used where a parameter or set of parameters is held constant while other parameters are varied. Although this works well for classical ANOVA, additional runs are needed if, as in this example, development of surrogate models is the ultimate goal. The use of surrogates for ANOVA analyses can significantly reduce the computational cost. To create the parameter population, a HaltonLeap deterministic sampling technique was chosen [16]. The Halton-Leap method creates multi-dimensional, uniformly distributed values between 0 and 1 , which are then converted to engineering parameter values within the 
bounds shown in Table 2. Numerous executions of nonlinear, dynamics simulations are not typically feasible because of time constraints. Thus upon completion of the 80 LS-Dyna simulations, a time-varying surrogate model was generated using the Extended Radial Basis Function (ERBF) approach developed by Mullur and Messac [17]. Although this example has relatively short execution times (less than 2 hours per simulation), many applications require substantially more time. Even for this application, while 1000 responses using this surrogate model are possible to complete in less than 20 minutes, 1000 FEM simulations would require 2 months. The variances of the resultant displacement were then computed using the Sobol method [18]. The Sobol method is suitable for a nonlinear design space where simple gradient computations at the parameter means may not be sufficient. The surrogate model in conjunction with the Sobol computation has been used to compute contributions of the three input parameters to the time-varying variance of the resultant displacement. The reader is reminded that the variance computations rely on the parameter ranges and distributions and the development of appropriate surrogate models.

\section{Discussion of Results}

For this application, the target behavior required the resultant displacement $(\mathrm{R})$ of Node B to reach a value of zero in the shortest possible time, with the minimum amount of overshoot and number of oscillations. Attainment of this goal would demonstrate that tape-spring designs exhibit both controlled and prescribed deployment behavior for space applications. The design process was divided into two phases. Phase A, based on two sets of 80 simulations, assessed the importance of the design parameters and selected ranges. Phase B focused on selecting a set of design parameters to produce the target behavior. To verify the final design, the selected parameters were used in LS-Dyna, and the response was compared to the target.

\section{A. Phase A: Assessing Parameter Importance}

For both OS and SS folding configurations, 80 simulations were executed. Figures $3 \mathrm{a}$ and $4 \mathrm{a}$ give the time histories for these simulations. The OS configuration, Fig. 3a, shows little variation during the folding (first 0.25 seconds) and considerable variation, primarily overshoot of the final position $(\mathrm{R}=0)$, during deployment (the final 0.25 seconds). The results for the $80 \mathrm{SS}$ simulations have been plotted in Figure $4 \mathrm{a}$. Unlike the OS folding cases, little overshoot of the target position exists for the SS folding simulations. However, considerable variation is evident during both the folding and deployment.

These 80-simulation sets were used to generate surrogate models (i.e., ERBF response surfaces). For each folding configuration and at each time step, a unique response surface is generated. Subsequently, the surrogate models were evaluated 1000 times for use in the Sobol variance computations. Figures $3 \mathrm{~b}$ and $4 \mathrm{~b}$ show the results of the variance computations for the OS and SS configurations, respectively. The bars at each time slice provide an indication of the contribution of the parameter to the total variance. For example, in Figure $3 \mathrm{~b}$ at the time of release $(\mathrm{t}=0.25 \mathrm{~s})$, nearly all of the variance can be attributed to the arc length parameter, indicated in red. Not all parameters showing a high contribution to the variance will result in a high correlation coefficient. However, for this case, the behavior was confirmed as the correlation coefficient between arc length and R (@0.25s) was 0.83. Variation in $\mathrm{R}$ at release can be attributed to the natural tendency of the tape to widen the distance between Nodes $\mathrm{A}$ and $\mathrm{B}$ to a minimum energy state, while maintaining the enforced end rotation. For the initial deployment, between 0.25 and 0.28 seconds, the thickness is the primary contributor to the variance. After 0.3 seconds, the variance is primarily controlled by the arc length and thickness. On the other hand, the SS folding has significant contributions from all three parameters throughout the folding and deployment (Fig. 4b).

The variance computations as a function of time provide insight into parameter importance. However, care must be exercised when one uses variance information in the design process since the variance is implicitly dependent on the range of the input parameters. Thus, if the parameter maxima and minima in Table 2 were changed, then the corresponding parameter contribution to the variance will also change. It was anticipated that one of the parameters could have been eliminated. However, based on variance analyses, all three parameters were retained.

When working with surrogate models, users should always be concerned about their adequacy. Surrogate model adequacy was assessed by the removal of the $i^{\text {th }}$ LS-Dyna solution from the solution set and the comparison of it to the surrogate prediction. In other words, the surrogate model did not contain the $i^{\text {th }}$ solution being evaluated. This approach was implemented because nonlinear FEM simulations are often computationally expensive. The removal process, depicted in Figure 5, can be performed with all 80 LS-Dyna solutions. The error (or accuracy) of the surrogate model was quantified by the computation of the root-sum-square (RSS) of the difference between the surrogate prediction and LS-DYNA time histories.

The resulting RSS error values for both folding configurations are shown in Figure 6 and arranged in descending order of magnitude. Note that the order of the RSS results in Figure 6 is not related to the order of the Halton-Leap 
sequence. Also, the error for the SS configuration is significantly higher than that for the OS configuration. High RSS SS error values are correlated to the inability of the response surface to capture the folding response. To illustrate the difference between simulation and surrogate model predictions, time histories for the $10^{\text {th }}, 30^{\text {th }}, 50^{\text {th }}$, and $70^{\text {th }}$ rank orders are provided in Figures 7 and 8. For the OS configuration, see Fig. 7, the surrogate model closely follows both the folding and the free deployment up to 0.3 seconds. However, after 0.3 seconds, significant differences are evident for both the $10^{\text {th }}$ and $30^{\text {th }}$ rank order, subplots (a) and (b), respectively. Figure 8 shows the complementary SS time history comparisons. Specifically, discrepancies with the folding response generated by the surrogate are evident for the $10^{\text {th }}$ simulation, subplot $8 \mathrm{a}$. Note that the $70^{\text {th }}$ simulation for each folding configuration is reasonable based on the qualitative comparison of time history traces. These qualitative comparisons when combined with the corresponding RSS error values provide insight as to a reasonable value to be expected for a measure of closeness to a target behavior. Essentially, an RSS value less that 0.3 could be considered reasonable.

As noted previously, these preliminary results were intended to provide insight as to regions of robust deployment. To that end, notional target bounds on the response were generated (Fig. 9). The notional bounds have been implemented to illustrate a method by which the time histories could be assessed. For those solutions within the target bounds, the corresponding parameters are provided in Figure 10 for both OS and SS configurations. To facilitate comparisons, the parameter values have been nondimensionalized by the transformation of their magnitudes such that the range was 0 to 1 with a mean of 0.5 . Intuitively, in this nondimensional space, parameter variations within a small band (i.e., less than the full range) tend to indicate dominant parameters that would produce a response within the bounds. Both of the folding configurations show a strong dependence on the arc length. Thus, high arc lengths are more desirable. Both of the folding configurations show a weak and likely insignificant dependence to the tape thickness. However, this lack of dependence could also be attributed to the notional bound definitions. Specifically, the variance contribution showed a strong contribution of thickness between 0.25 and 0.28 seconds (Fig. 3b). However, all of the OS simulations fell within the notional bounds. Thus, the dependence would not be identified based on the results in Figure 10. Additionally, the targeted behavior for the OS folding is more likely to occur for tapes with a smaller radius. For the SS configuration simulations that fell outside the notional folding bounds (up to $0.25 \mathrm{~s}$ ), the arc lengths were less than the mean. However, not all simulations with arc lengths less than the mean exhibited this response. An exhaustive review of the results did not provide additional insights as to the relative interaction of the input parameters to generate responses within the bounds. Ultimately, the tape springs are frequently paired in most applications. Thus, the desired deployment behavior is more likely for designs with larger arc lengths.

\section{B. Phase B: Parameter Selection Process to Produce Target Response}

The SS configuration can exhibit a somewhat erratic behavior for some of the small radii cases because of insufficient specification of the end conditions. Simply enforcing an end rotation is not sufficient for this folding configuration and these parameter ranges. The erratic behavior during folding resulted in two $90^{\circ}$ folds instead of one $180^{\circ}$ fold. As the two $90^{\circ}$-fold hinge is not a likely design configuration, the SS configuration study will be discontinued in Phase B.

The parameter space for the OS configuration was also significantly reduced based on the Phase A results to focus on regions more likely to produce robust designs. Specifically, the nondimensional radius was halved to span 0 to 0.5 (reference Fig. 10) while the arc length and thickness ranges were also halved to span 0.5 to 1.0. Thus, the total design space was one-eighth of that originally studied. Forty-five additional LS-Dyna OS simulations were executed, and the results were added to the existing set of 80 to generate the Phase B surrogate models. The focus for these subsequent simulations was a parameter set where the surrogate model exhibited the desired behavior. As for the original 80 simulations, the adequacy of the surrogate model was assessed by the ability of the model to predict the FEM simulation responses. Only 40 solutions of the 125 -simulation set fell within the revised parameter bounds. In Figure 11, the RSS error for these 40 simulations has been plotted with the original OS data from Figure 6. The RSS error has been approximately halved. Figure 12 provides a sampling of the surrogate versus simulation time history comparisons that used the reduced parameter space. Specifically, comparisons for the $5^{\text {th }}, 15^{\text {th }}, 25^{\text {th }}$, and $35^{\text {th }}$ rank order simulations are provided. It is understood that surrogate models can be used to generate a wealth of design data at a significantly reduced computational cost. However, the price is less accuracy. The accuracy of the surrogate model, as reflected in the RSS error computation, was deemed sufficient to continue with the design process illustration.

For design, a target resultant-displacement time history was generated, and the RSS of the difference between the ERBF model prediction and the target response was computed for 1000 Monte Carlo parameter sets. At this point the ERBF model could have been used with a nonlinear optimization scheme to determine the parameter set with the smallest RSS error. Instead, the parameter set that produced the smallest error from the 1000 ERBF 
predictions was selected (i.e, radius $=0.0110 \mathrm{~m}$, arc length $=76.9^{\circ}$, thickness $=1.069 \times 10^{-4} \mathrm{~m}$ ). To confirm the design, the parameter set producing the smallest RSS error was used in a final LS-Dyna simulation. Figure 13 shows the results for the LS-Dyna simulation, the target response, and the ERBF predictions. The RSS error for the simulation is 0.17 while that for the ERBF is 0.16 when compared to the target response. As expected, results from the LS-Dyna model follow the target response reasonably well.

\section{Concluding Remarks}

Acceptance of new deployable structures architectures and concepts require validated design methods to minimize the expense involved with technology validation and flight testing. This paper explores the implementation of probabilistic methods for a nonlinear, structural, dynamic application, namely the free deployment of a strain-energy hinge. The simulation of the folding and free-deployment of a simple tape-spring was automated with a combination of MATLAB scripts and LS-Dyna simulations. A surrogate model was generated to provide time history responses, and a metric was established to evaluate the surrogate model accuracy. The results were assessed by examination of the free-end resultant displacement during both folding and free deployment for two folding configurations: opposite-sense (OS); and same-sense (SS). Results show that:

1. The deployments were numerically stable over the relatively wide range of the geometric parameters varied in the probabilistic analysis.

2. The surrogate model was successfully used in the design process to determine parameter values that produced a controlled and prescribed behavior.

In summary, the application of probabilistic analysis for evaluation of a tape-spring hinge has shown promise as a means of assessing strain-energy joints for lightweight space applications involving more complex designs.

\section{References}

[1] Footdale, J. N. and Murphey, T. W., "Structural Design of a CubeSat-Based Diffractive Optic Telescope," Proceedings of the AIAA/ASME/ASCE/ASC Structures, Structural Dynamics and Material Conference, AIAA Paper 2011-1729, Denver CO, 4-7 April 2011.

[2] Dorsey, John T., Collins, Timothy J., Moe, Rud V., and Doggett, William R., "Framework for Defining and Assessing Benefits of a Modular Assembly Design Approach for Exploration Systems," Proceedings of Space Technology and Applications International Forum (STAIF-2006), Albuquerque NM, 12-16 Feb. 2006.

[3] Gardner, J. R., et al., "The James Webb Space Telescope," Space Science Reviews, Vol. 123, No. 4, April 2006, pp. 485-606.

[4] Lyle, K. H., Stockwell, A. E., and Hardy, R. C., "Application of Probability Methods to Assess Crash Modeling Uncertainty," AIAA Journal of Aircraft, Vol. 44, No. 5, Sept.-Oct. 2007, pp. 1568-1573.

[5] Mobrem, M., and Adams, D. S., "Analysis of the Lenticular Jointed MARSIS Antenna Deployment," Proceedings of the AIAA/ASME/ASCE/ASC Structures, Structural Dynamics, and Material Conference, AIAA Paper 2006-1683, Newport RI, 1-4, May 2006.

[6] Seffen, K. A., and Pellegrino, S., "Deployment dynamics of tape springs," Proc. R. Soc. Lond. A, Vol. 455, No. 1983, 1999, pp. 1003-1048.

[7] Mallickarachchi, H. M. Y. C. and Pellegrino, S., "Optimized Designs of Composite Booms with Integral Tape-Spring Hinges," Proceedings of the AIAA/ASME/ASCE/ASC Structures, Structural Dynamics, and Material Conference, AIAA Paper 2010-2750, Orlando FL, 12-15 April 2010.

[8] Mallickarachchi, H. M. Y. C. and Pellegrino, S., "Design and Validation of Thin-Walled Composite Deployable Booms with Tape-Spring Hinges," Proceedings of the AIAA/ASME/ASCE/ASC Structures, Structural Dynamics, and Material Conference, AIAA Paper 2011-2019, Denver CO, 4-7 April 2011.

[9] Olson, G. M., Murphey, T., and Thomas, G., "Free Deployment Dynamics of a Z-Folded Solar Array," Proceedings of the AIAA/ASME/ASCE/ASC Structures, Structural Dynamics, and Material Conference, AIAA Paper 2011-1730, Denver, CO, 4-7 April 2011.

[10] Straubel, M., Block, J., Sinapius, M., and Huhne, C., "Deployable Composite Booms for Various Gossamer Space Structures," Proceedings of the AIAA/ASME/ASCE/ASC Structures, Structural Dynamics, and Material Conference, AIAA Paper 2011-2023, Denver, CO, 4-7 April 2011. 
[11]Kwok, K. and Pellegrino, S., "Viscoelastic Effects in Tape-Springs," Proceedings of the AIAA/ASME/ASCE/ASC Structures, Structural Dynamics, and Material Conference, AIAA Paper 2011-2022, Denver, CO, 4-7 April 2011.

[12]Rose, Geoffrey K., "Investigation of the Modeling and Simulation Capability of the Folding and Deployment of a Tape-springs Using LS-Dyna," To be published as NASA Technical Memorandum.

[13]Fischer, A., "Bending Instabilities of Thin-Walled Transversely Curved Metallic Strips," Report: CUED/D-STRUCT/TR 154 Cambridge University, 1995.

[14] Annett, M.S. and Horta, L.G., "Comparison of Test and Finite Element Analysis for Two Full-Scale Helicopter Crash Tests," 52nd AIAA/ASME/ASCE/AHS/ASC Structures, Structural Dynamics, and Materials Conference, Denver, CO, 4-7 April 2011.

[15]LS-Dyna Keyword User's Manual, Version 971, May 2007.

[16] Halton, J.H., "On the Efficiency of Certain Quasi-Random Sequences of Points in Evaluating MultiDimensional Integrals," Numerische Mathematik, Vol. 2, 1960, pp. 84-90.

[17] Mullur, A. A. and Messac, A., "Extended Radial Basis Functions: More Flexible and Effective Metamodeling," Proceedings of the $10^{\text {th }}$ AIAA/ISSMO Multidisciplinary Analysis and Optimization Conference, AIAA Paper 2004-4573, Albany, NY, 30 August-1 September 2004.

[18] Sobol, I.M., Tarantola, S., Gatelli, D., Kucherenko, S.S., and Mauntz, W., "Estimating Approximation Error When Fixing Unessential Factors in Global Sensitivity Analysis," Reliability Engineering and System Safety, Vol. 92, 2007, pp. 957-960.

Table 1. Deterministic tape parameters.

\begin{tabular}{|c|c|}
\hline Parameter & Value \\
\hline Density & $8400 \mathrm{~kg} / \mathrm{m}^{3}$ \\
\hline Young's Modulus & $1.31 \times 10^{11} \mathrm{~N} / \mathrm{m}^{2}$ \\
\hline Poisson's Ratio & 0.3 \\
\hline Length & $0.2 \mathrm{~m}$ \\
\hline
\end{tabular}

Table 2. Design parameters incorporated in PA.

\begin{tabular}{|c|c|c|}
\hline Parameter & Minimum & Maximum \\
\hline Radius, $m$ & 0.010 & 0.015 \\
\hline Arc length, radians (degrees) & $0.78\left(45^{\circ}\right)$ & $1.50\left(87.5^{\circ}\right)$ \\
\hline Thickness, $m$ & $0.75 \times 10^{-4}$ & $1.25 \times 10^{-4}$ \\
\hline
\end{tabular}




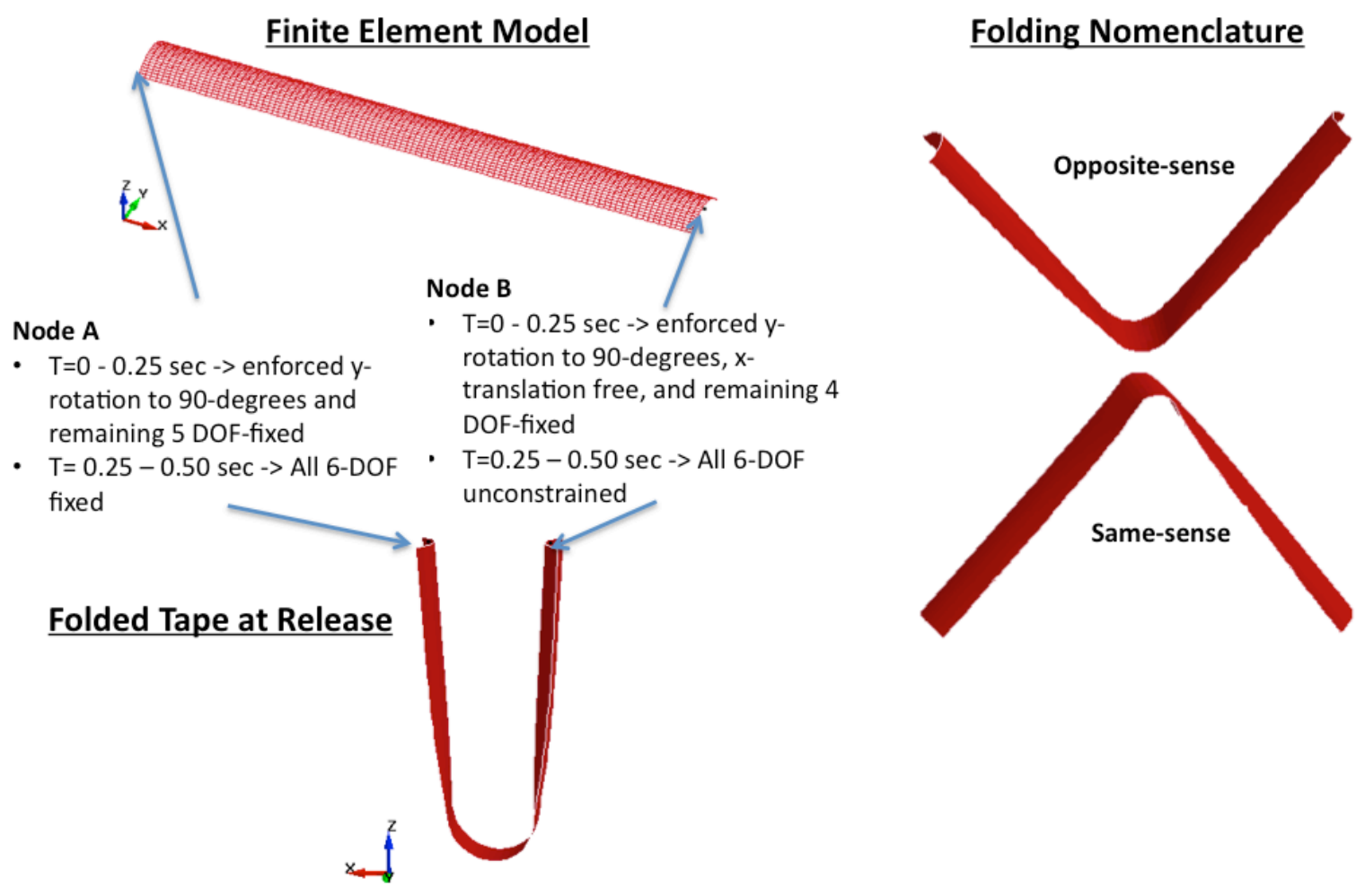

Figure 1. Finite element schematic with tape-folding nomenclature.

Tape Cross-section

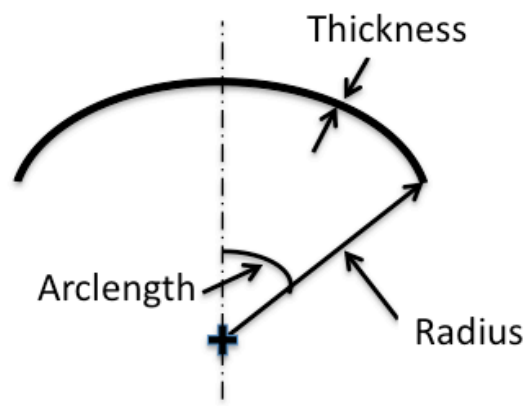

Figure 2. Schematic of tape cross-section with uncertain parameters identified. 

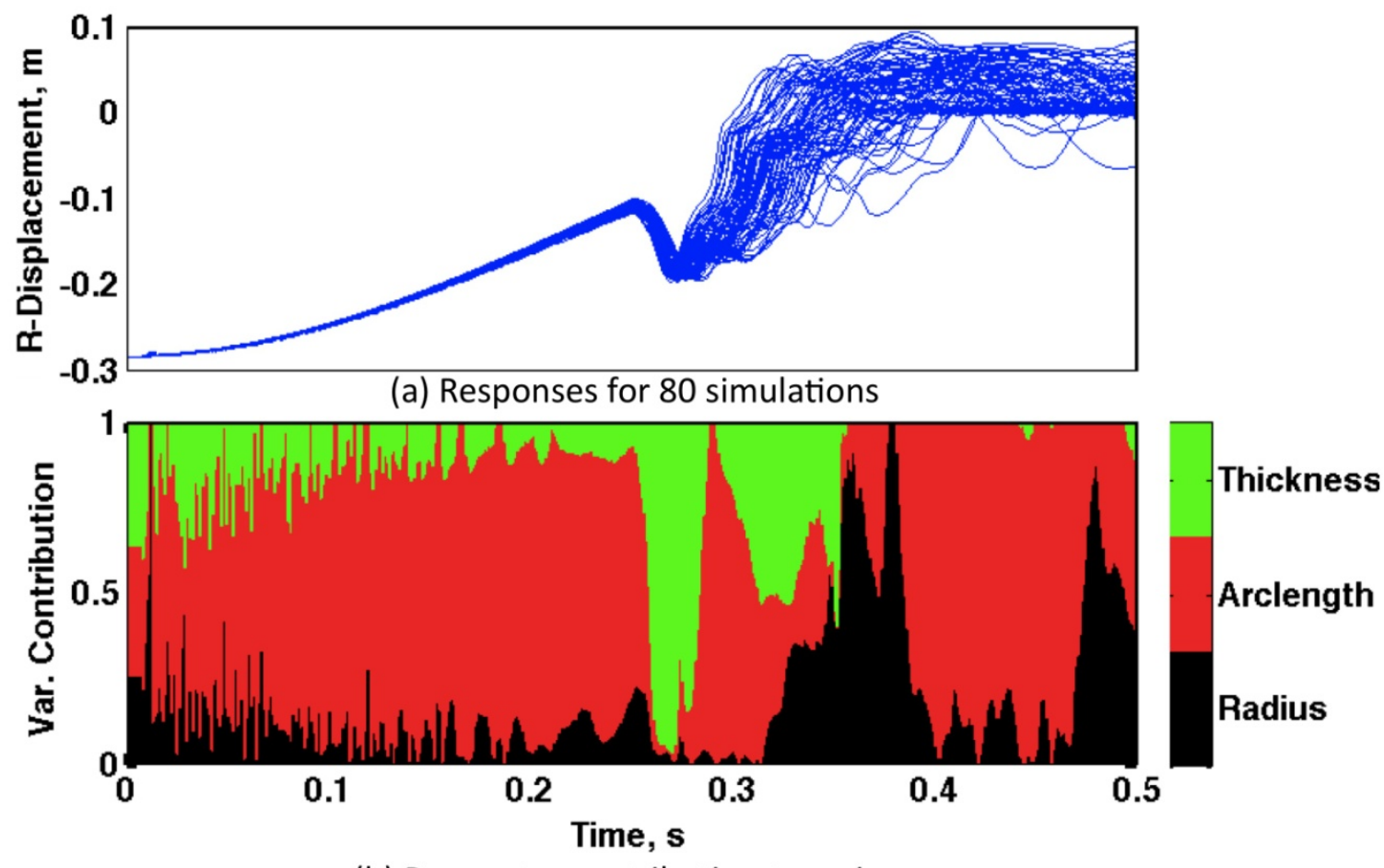

(b) Parameter contribution to variance

Figure 3. OS folding configuration: (a) $R$ time histories for 80 simulations; (b) Contribution to variance of $R$.

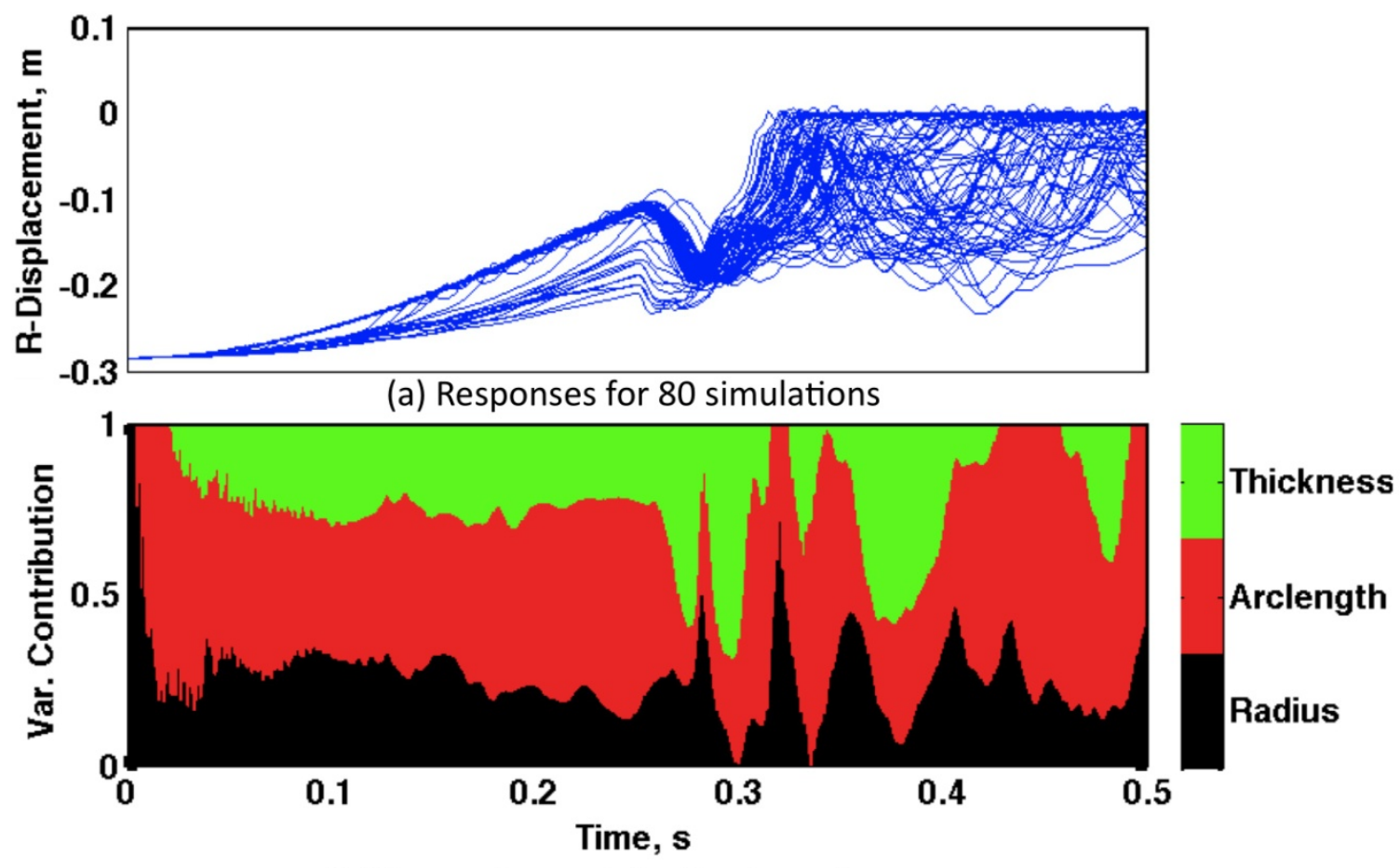

(b) Parameter contribution to variance

Figure 4. SS folding configuration: (a) $R$ time histories for 80 simulations; (b) Contribution to variance of $R$. 


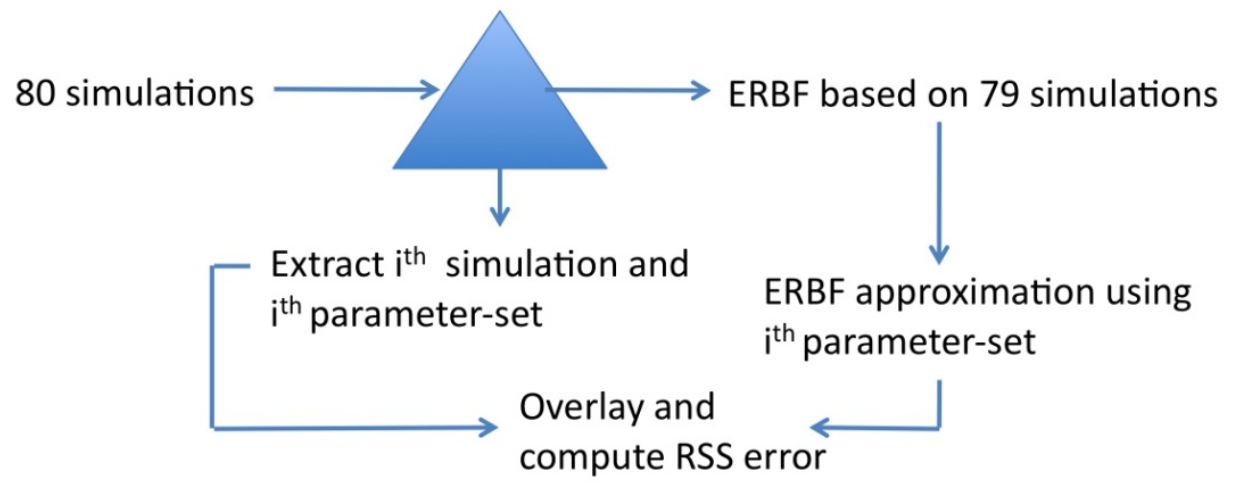

Figure 5. Flow-chart for response surface adequacy computation.

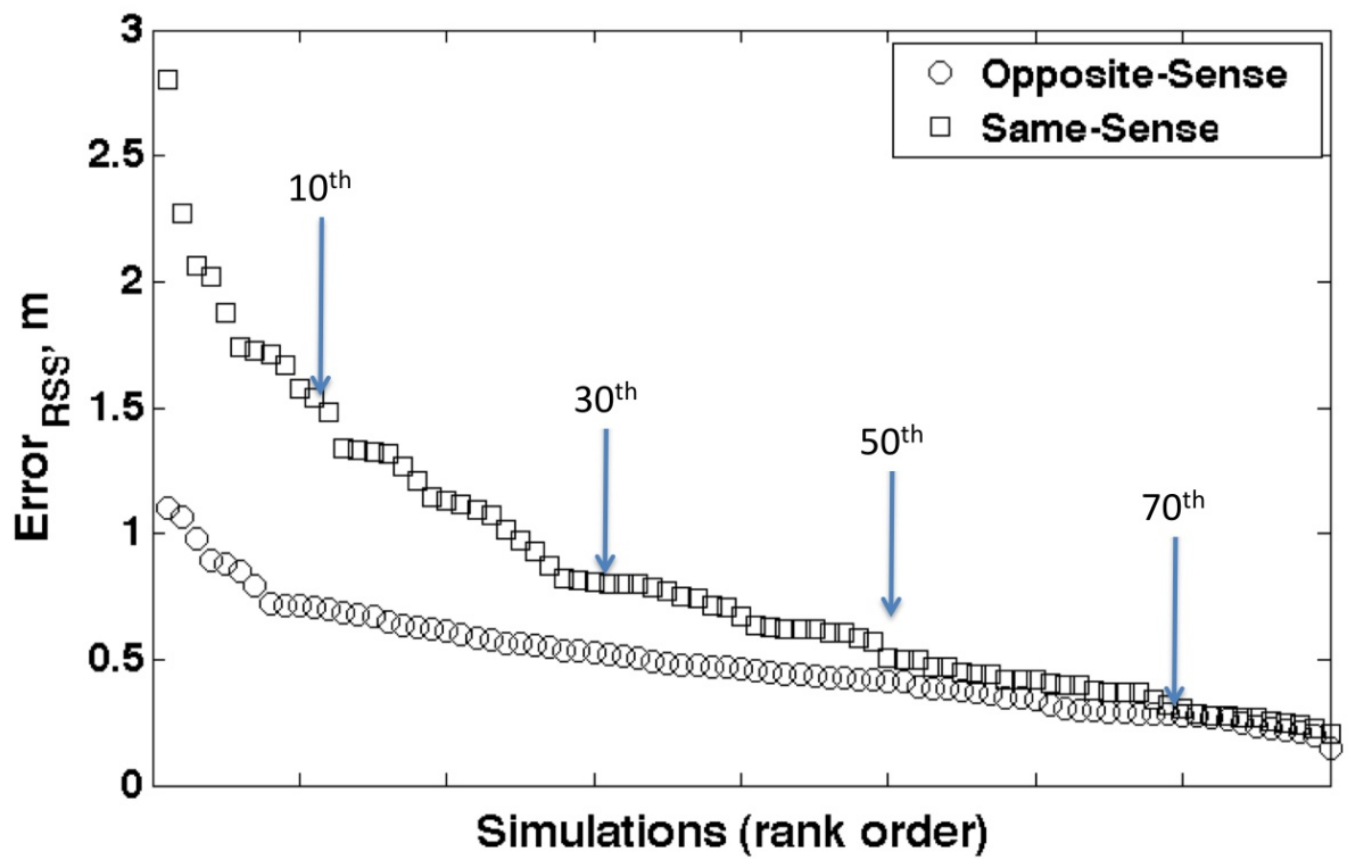

Figure 6. RSS error for Phase A surrogate models when compared to simulations. 

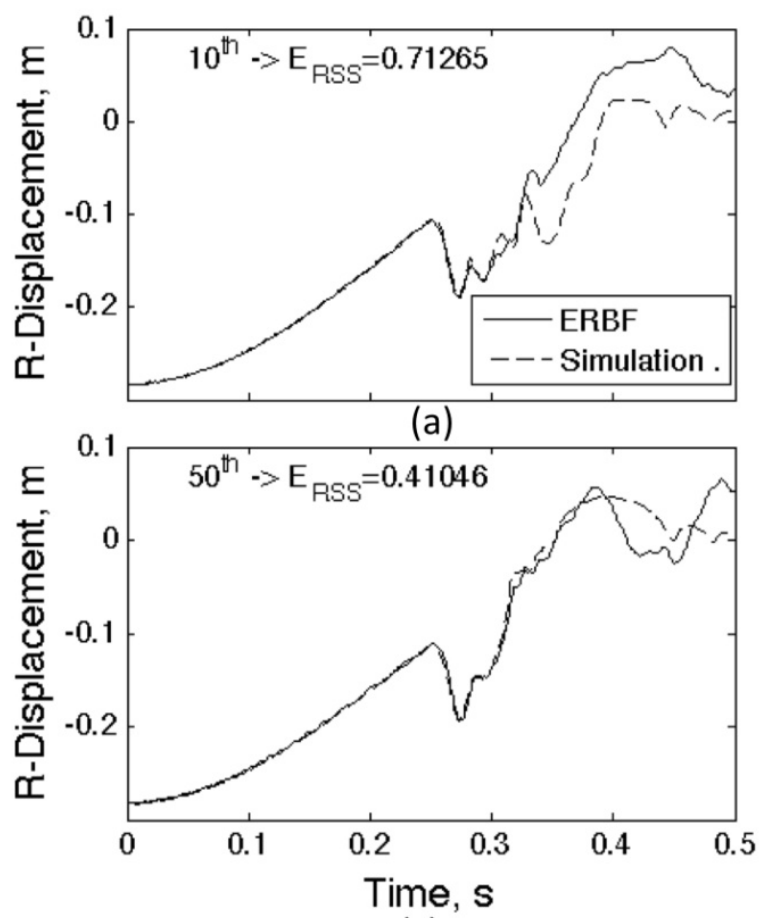

(c)
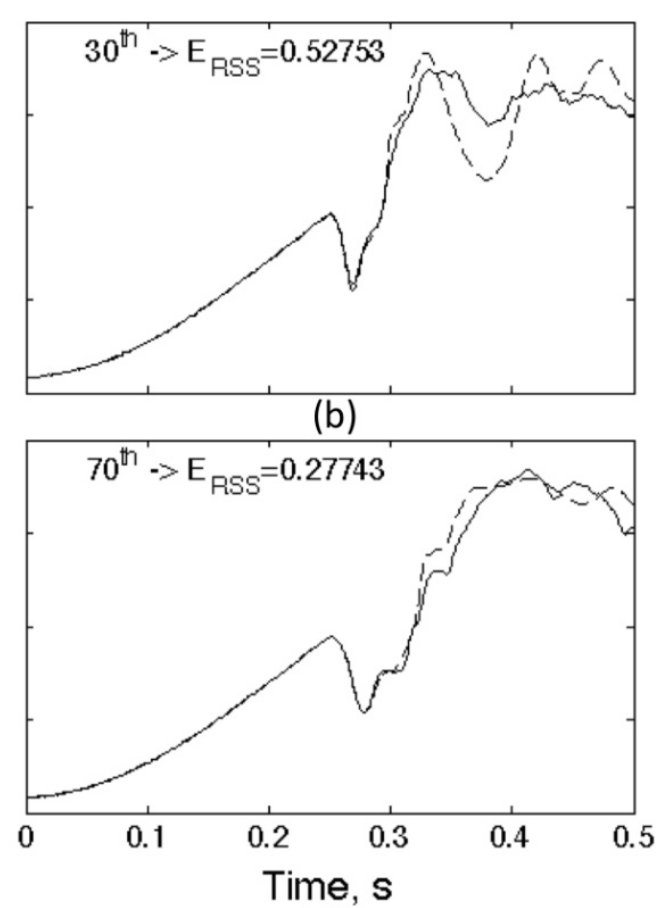

(d)

Figure 7. OS: Sample time history comparisons of Phase A surrogate models and simulations.

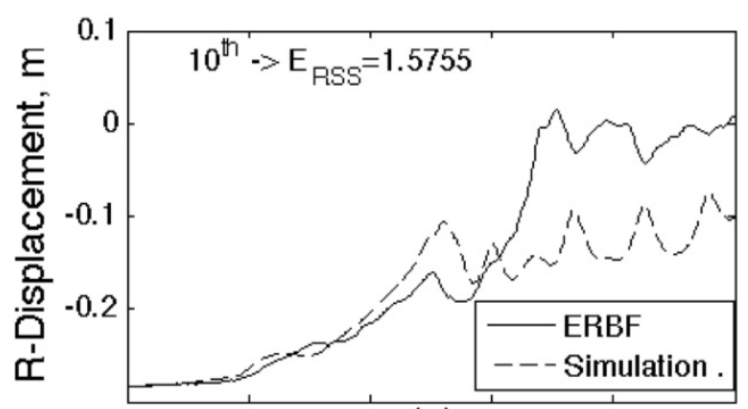

(a)

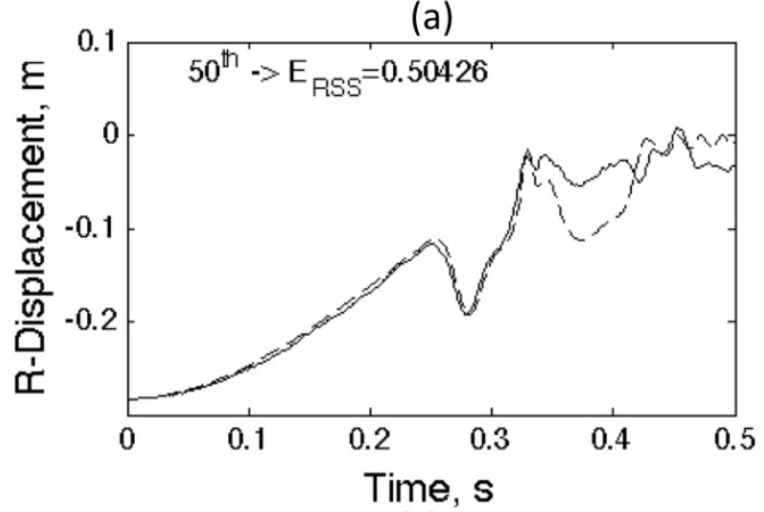

(c)

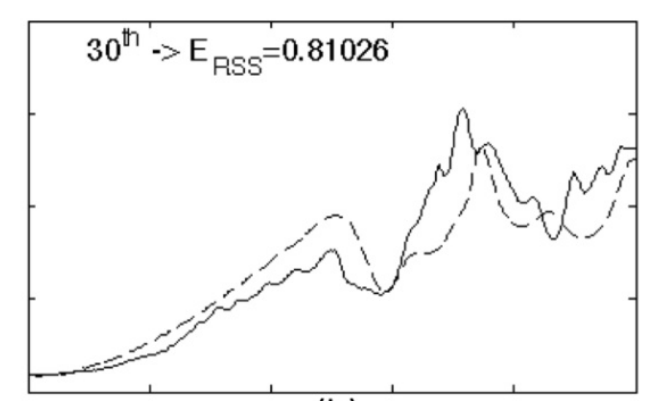

(b)

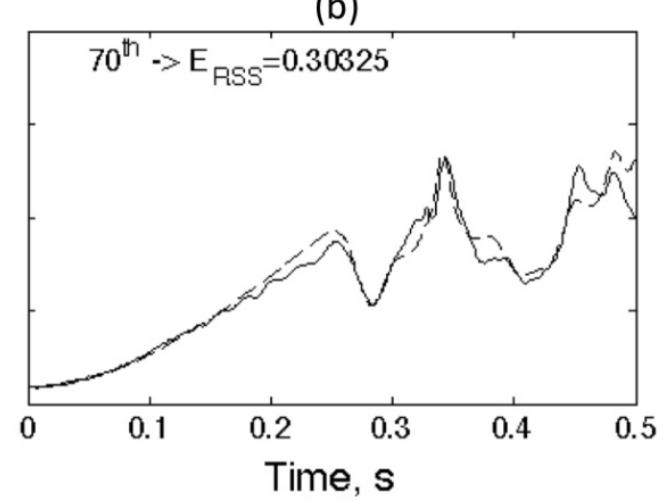

(d)

Figure 8. SS: Sample time history comparisons of Phase A surrogate models and simulations. 

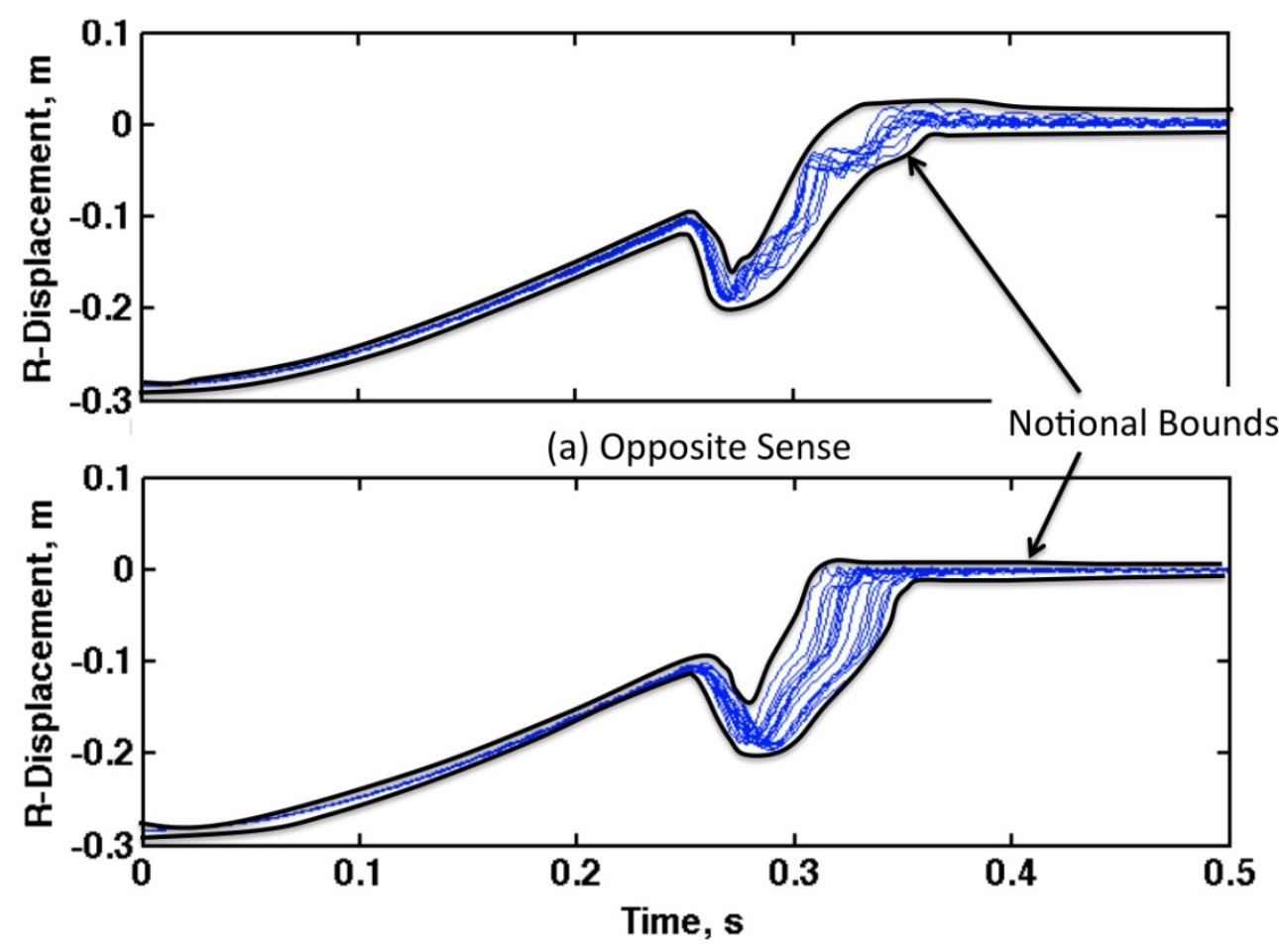

(b) Same Sense

Figure 9. Phase A simulations falling within notional bounds: (a) OS; (b) SS configuration.

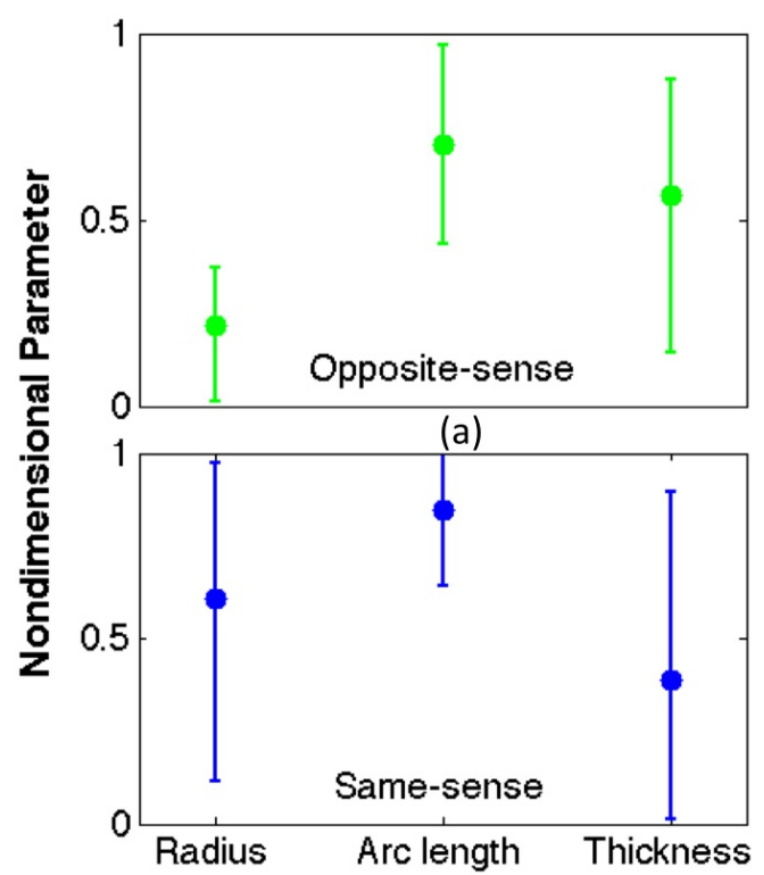

(b)

Figure 10. Parameter distributions for simulations falling within notional bounds provided in Figure 10: (a) OS; (b) SS configuration. 


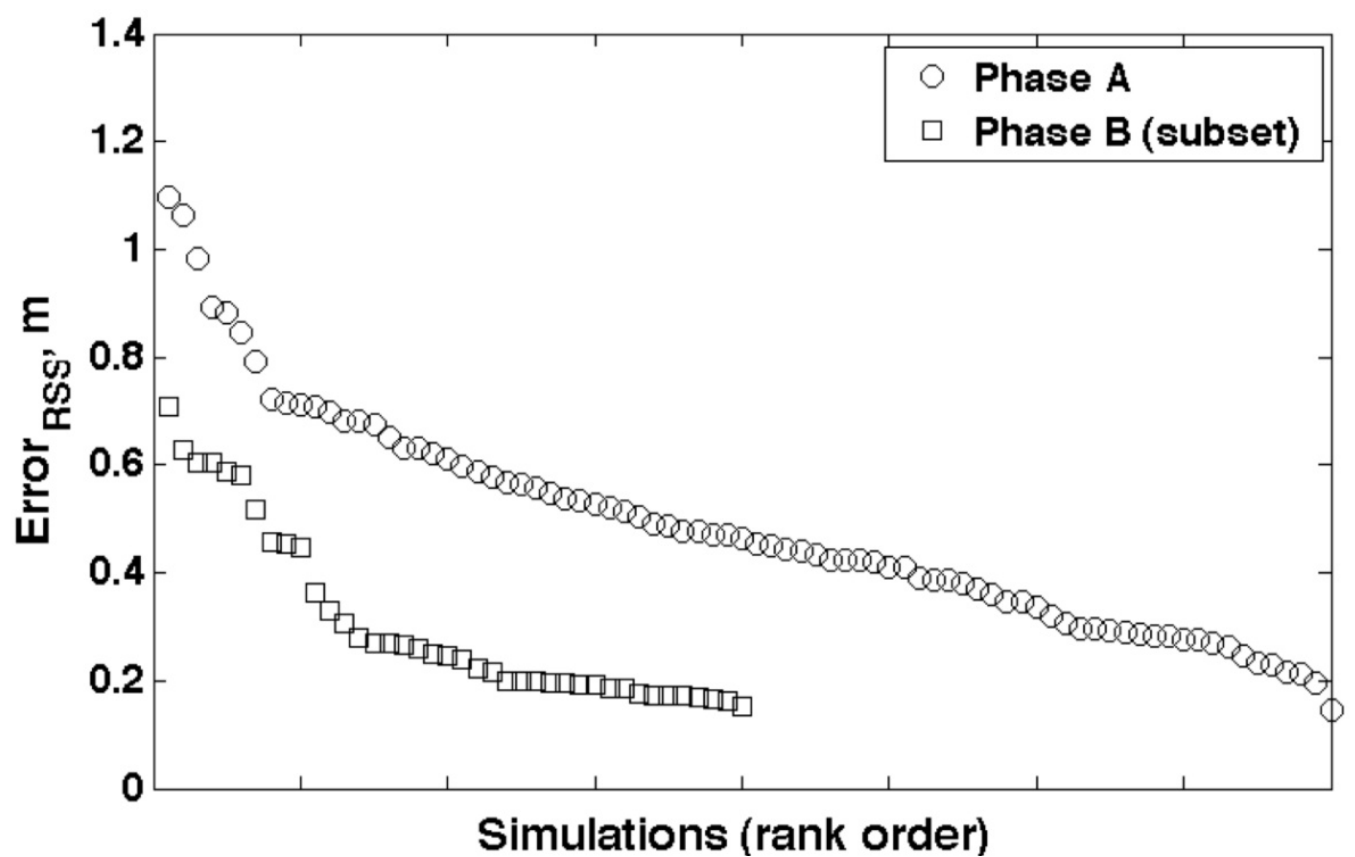

Figure 11. RSS error for Phase A surrogate model and Phase B surrogate model.

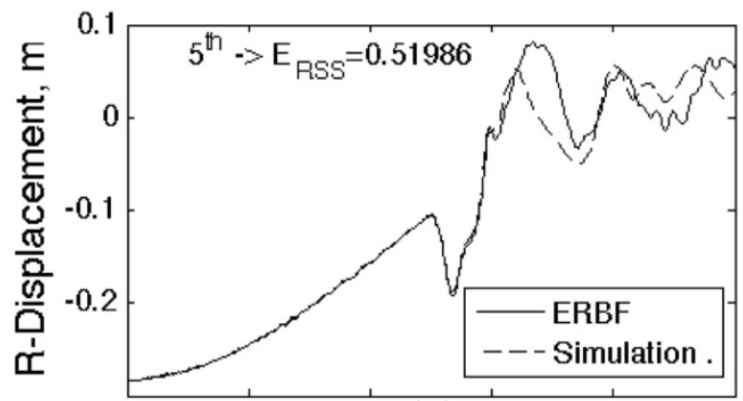

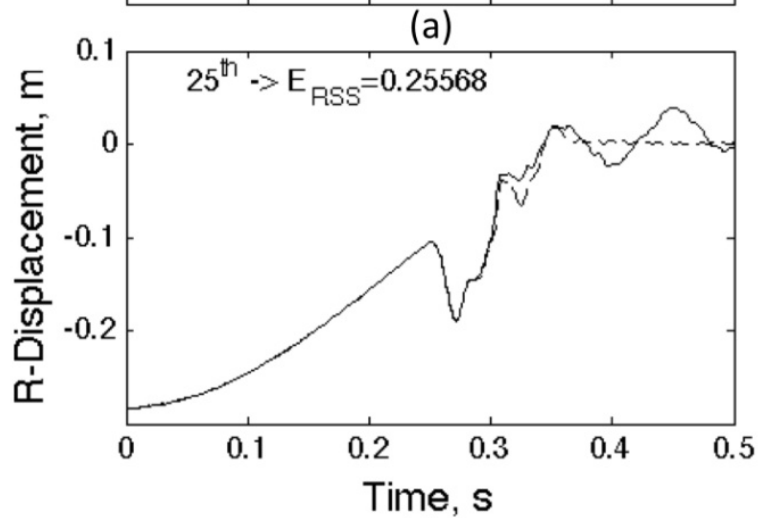

(c)

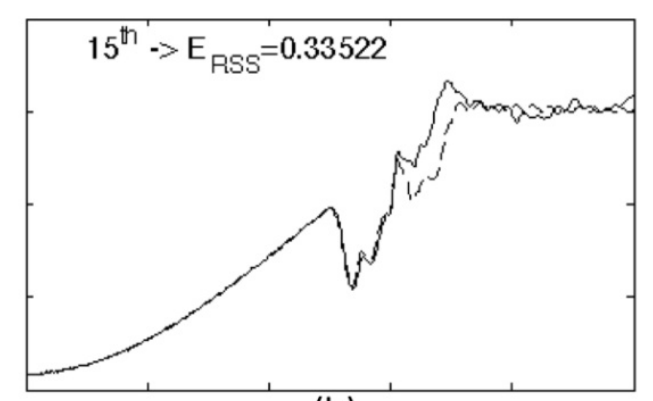

(b)

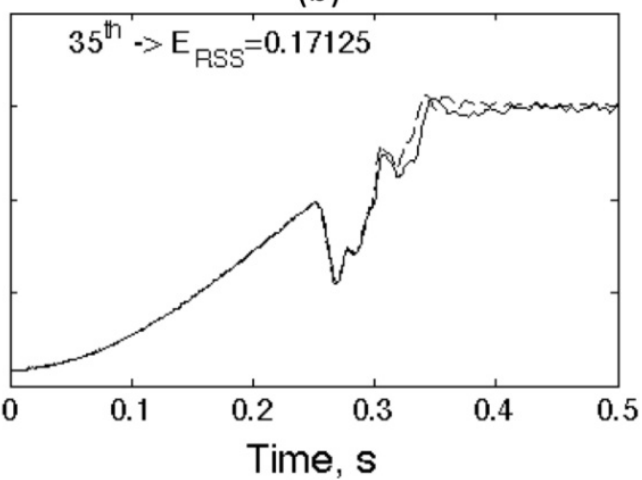

(d)

Figure 12. Sample time history comparisons of Phase B surrogate models and simulations. 


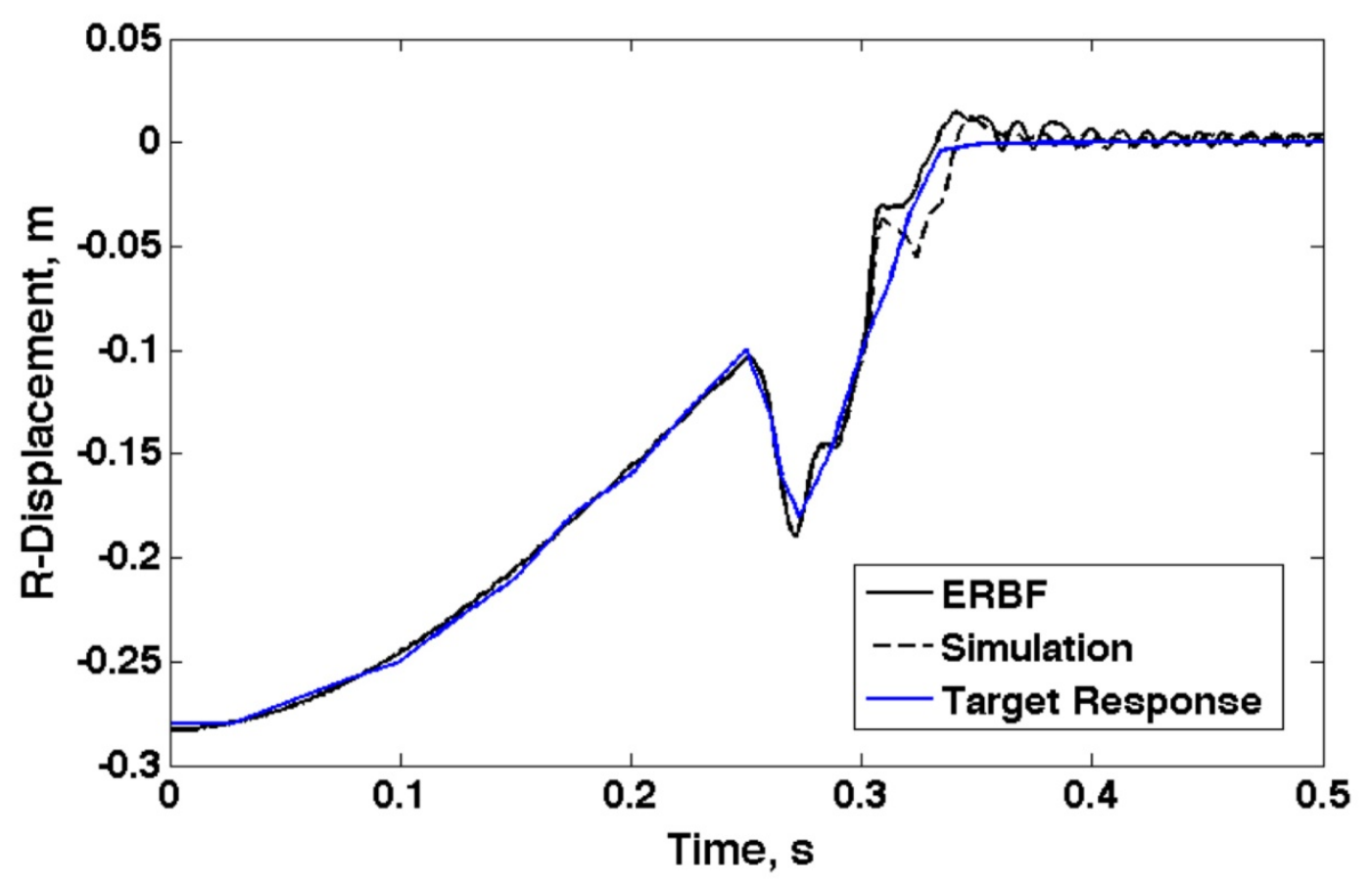

. Figure 13. OS: Comparisons of best-fit response surface results. 